
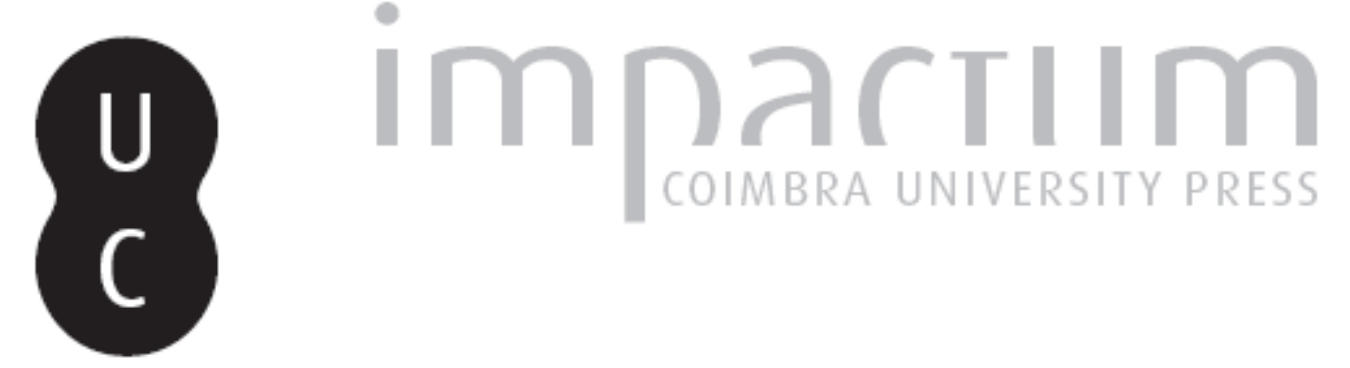

\title{
[Recensão a] F. MUCCIOLI, La storia attraverso gli esempi. Protagonisti e interpretazioni del mon do greco in Plutarco
}

\author{
Autor(es): $\quad$ González González, Marta \\ Publicado por: International Plutarch Society \\ URL \\ persistente: \\ URI:http://hdl.handle.net/10316.2/36377 \\ DOI: \\ DOI:http://dx.doi.org/10.14195/0258-655X_9_13 \\ Accessed : $\quad$ 26-Apr-2023 13:39:04
}

A navegação consulta e descarregamento dos títulos inseridos nas Bibliotecas Digitais UC Digitalis, UC Pombalina e UC Impactum, pressupõem a aceitação plena e sem reservas dos Termos e Condições de Uso destas Bibliotecas Digitais, disponíveis em https://digitalis.uc.pt/pt-pt/termos.

Conforme exposto nos referidos Termos e Condições de Uso, o descarregamento de títulos de acesso restrito requer uma licença válida de autorização devendo o utilizador aceder ao(s) documento(s) a partir de um endereço de IP da instituição detentora da supramencionada licença.

Ao utilizador é apenas permitido o descarregamento para uso pessoal, pelo que o emprego do(s) título(s) descarregado(s) para outro fim, designadamente comercial, carece de autorização do respetivo autor ou editor da obra.

Na medida em que todas as obras da UC Digitalis se encontram protegidas pelo Código do Direito de Autor e Direitos Conexos e demais legislação aplicável, toda a cópia, parcial ou total, deste documento, nos casos em que é legalmente admitida, deverá conter ou fazer-se acompanhar por este aviso.

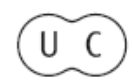


Ploutarchos, n.s.

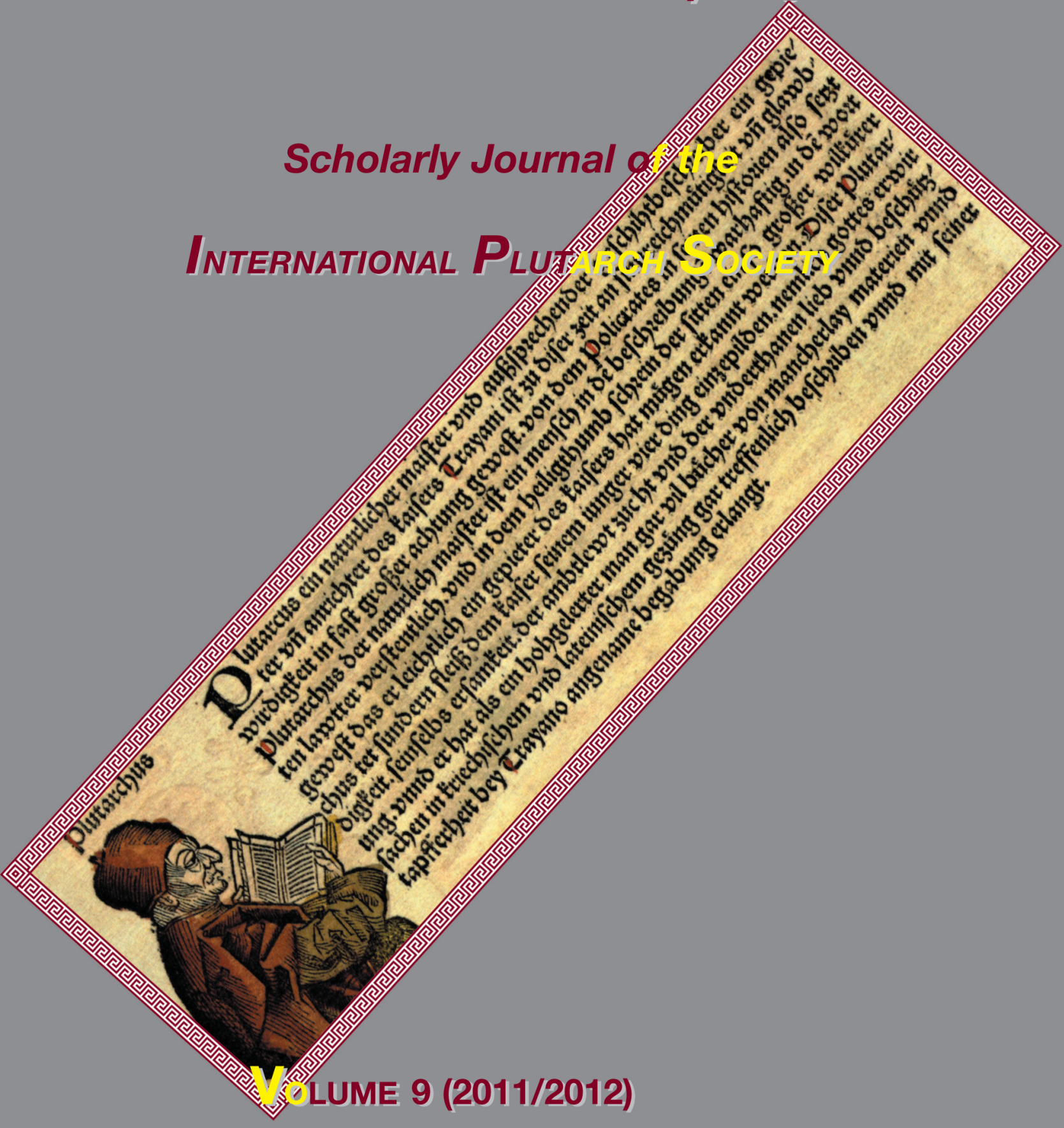

University of Málaga (Spain) Utah State University, Logan, Utah (U.S.A.) 
sketch of Greece's conflict-ridden history in Flam. 11.3-7 (V. refers to it on p. 203) the named culprits are "Agesilauses, Lysanders, Niciases, Alcibiadeses" - in the plural form! Plutarch is quite prepared to view Alcibiades as agressively unique ${ }^{1}$ and larger than life ("Greece could not endure two Alcibiadeses", Alc. 16.8, cf. Lys. 19.5), but at the end of the day his idea of the hero is still paradigmatic and hence unified despite of, or maybe thanks to, his paradoxicalness.

\section{AleXeI V. ZAdorojnYI \\ University of Liverpool}

F. Muccioli, La storia attraverso gli esempi. Protagonisti e interpretazioni del mondo greco in Plutarco, Milano-Udine, Mimesis Edizioni, 2012, 350 pp. ISBN 978-88-5750-934-1.

Federicomaria Muccioli plantea en una clara introducción el objetivo de su ensayo: poner a prueba la idea de si Plutarco tenía una visión propia de la historia, una metodología histórica susceptible de ser analizada en el conjunto de su vasta obra por encima de la variedad de formas literarias empleadas. Plantea Muccioli como premisas que, pese a la extensión de la producción del queronense, es posible hablar de una unidad del Corpus Plutarcheum y que, entre los múltiples niveles de lectura a los que se presta su obra, el de su metodología histórica está todavía necesitado de un estudio de conjunto.

El capítulo 1, "Exempla storici e biografie" (pp. 21-89), analiza en detalle, a través de seis epígrafes, diferentes aspectos relacionados con el método de composición de Plutarco. Son muchos los problemas que se abordan de manera crítica; para empezar, el de las citas. Muccioli se pronuncia en contra del prejuicio (basado en una referencia del autor a su retiro en Queronea en Demosth. 1-2) de que apenas ninguna de ellas es directa y considera, en cambio, que el autor tuvo acceso a muchas de las fuentes que cita. Considera, en relación con el recurso a hypomnemata, que se trataba de una especie de "apuntes personales" (para esta idea cita a Van der Stock), un conjunto de reflexiones críticas probablemente influidas por su formación retórica. También se aborda en este capítulo la cuestión de los ejemplos, personajes y episodios elegidos por Plutarco tanto en las Vidas como en Moralia. Para el autor, las Vidas son un intento de explicar la historia griega y latina a través del recorrido ético, más que político, de sus más significativos protagonistas; siendo el valor educativo y ético el dominante, los episodios y anécdotas son las mismas que en el resto del Corpus Plutarcheum ${ }^{1}$. Hay un aspecto interesante que señala Muccioli, y es que en la obra de Plutarco se ha superado la polémica de épocas precedentes sobre la superioridad de los modelos griegos o los romanos. El spatium geográfico es también analizado para concluir que prima la perspectiva atenocéntrica y espartanocéntrica, con grandes concesiones a Beocia y sobre todo Tebas, y el poco interés por la periferia, con la puntual excepción de Egipto en De Iside

1 To Aristotle, a story of "what Alcibiades did or suffered" (Poet. 1451b9-10) famously exemplifies a particular (kath' hekaston) rather than universal tale. For "deep" intertexuality between Aristotle's passage and Plutarch's Life, see T. Duff, Plutarch's Lives: Exploring Virtue and Vice (Oxford 1999), 227.

$1 \mathrm{Al}$ ejemplificar sobre cómo Plutarco emplea a conveniencia los datos de los que dispone acerca de los personajes biografiados, descubrimos uno de los pocos errores del estudio de Muccioli (explicable, dado el volumen de la obra de Plutarco, abordada aquí en conjunto): la afirmación (p. 34) de que en la Vida de Dión Plutarco no menciona el hecho de que éste le costeó a Platón un coro, algo que sí hace el queronense en Dion 17.5. 
et Osiride. Otro aspecto que se aborda en este interesante primer capítulo es el del "lector ideal", el destinatario de la obra de Plutarco que, dice Muccioli, sería el grupo de los pepaideumenoi, lectores integrados en el sistema político y administrativo romano y capaces de orientarse a través de las referencias y citas tan frecuentes en la obra de este autor. Un asunto complejo es el del modelo de la obra plutarquea, entre historiografía y biografía, para el que suele citarse el famoso pasaje de la Vida de Alejandro (que por conocido no repetimos aquí). Más allá del valor relativo de las palabras de Plutarco en ese contexto, propone el autor de este ensayo que el queronense "renuncia a la historia sólo como género literario de narración extensa, no como indagación histórica" (p. 56) y que, al igual que Hecateo, Heródoto o Tucídides, tenía que atender a una deontología profesional: su criterio fue, como el de sus predecesores, el de lo verosímil. Muccioli atiende también a las fuentes menores, como Éforo, Teopompo, Timeo o Filarco y señala también que si la historiografía y propaganda puramente local está en general ausente de las biografías, es, en cambio, ampliamente utilizada en obras especializadas y eruditas como $\mathrm{Mu}$ lierum virtutes o Quaestiones Graecae. Finalmente, el epígrafe último de este primer capítulo se centra en el recurso a la autopsía, empleado por Plutarco para hablar de lugares, campos de batalla, tumbas, sobre todo de Atenas, pero también de Esparta, Beocia y Delfos, una autopsía que, en el caso de la mención de inscripciones, no siempre es fácil determinar si realmente las ha visto $o$ las cita a partir de fuente indirecta.

En el capítulo 2, "Tra mito e storia arcaica" (pp. 91-130), el autor aborda el espacio difuso entre mito e historia antigua a través de unos ejemplos muy acertadamente elegidos. Se parte del hecho de que Plutarco, como cualquier otro griego erudito, tiene en la tradición épica una fuente fundamental de su paideia y utiliza los exempla míticos siempre que le parece oportuno. Comienza Muccioli analizando la figura paradigmática de Heracles, cuya defensa hace Plutarco en clara polémica con Heródoto. Para el de Queronea se trata un héroe-dios tebano, el primer personaje de la historia griega merecedor de una biografía, paradigma fundador de la grecidad del que desciende el propio Alejandro. En sus referencias a Heracles, Plutarco racionaliza algunos de los episodios de su vida (los monstruos a los que se enfrentó eran tiranos injustos y brutales) e intenta acomodar otros al principio de verosimilitud. En cuanto a la Vida de Teseo, el héroe ateniense por excelencia era una opción ineludible. Llama la atención Muccioli sobre el hecho de que al hablar de su pareja romana, Rómulo, el autor obvia las tradiciones ligadas a la fundación de Roma, probablemente para evitar polémicas sobre su origen greco-troyano. Teseo, señala también Muccioli, constituye la única recreación plutarquea del pasado ateniense hasta Solón (tratado en la correspondiente Vita y en Septem sapientium convivium). Con respecto a Esparta, su pasado se interpreta a través de Licurgo: "La elección de Plutarco se explica teniendo en cuenta la tradición y un encuadramiento de la historia espartana, que a sus ojos es mucho más lineal que la historia ateniense. Si ésta aparecía fragmentada, sin un auténtico personaje que coagulara las dinámicas políticas y sociales de la edad arcaica hasta el siglo IV, el mundo espartano, en cambio, es visto como una degeneración respecto de la perfección licurguea" (p. 118). Finalmente, en su reconstrucción del pasado griego, también alude a las colonizaciones, aunque incurriendo en el mismo error de Tucídides de considerar el movimiento como un continuum, confundiendo primera y segunda colonización.

Tras la historia de corte mítico, la historia de la edad clásica: el capítulo 3, 'L'età del 
classico nella storia greca" (pp. 131-192) se centra en los grandes momentos, como las Guerras Persas. Señala Muccioli que la perspectiva de Plutarco es en este caso atenocéntrica (Temístocles, Arístides, Cimón) y deja en un segundo plano a los protagonistas espartanos, como demostraría la ausencia de una Vita de Pausanias, o de Leónidas, o de Milcíades, personajes que conoce bien y que aparecen en diferentes pasajes del resto de la obra plutarquea. Un lugar destacadísimo ocupa la Vida de Pericles, el personaje que encarna (para Plutarco, para Winckelmann, para tantos) el momento cumbre de la cultura griega. Es interesante la observación de Muccioli acerca de un posible valor funcional de esta Vita que se podría sumar a los muchos otros que se le reconocen: "La representación de la Atenas periclea como el momento clásico, con un valor atemporal, puede también ser leida, entre líneas, en relación con la celebración de Roma no sólo como corazón de la ecumene sino también como centro cultural y artístico. Una superioridad que no está subrayada abiertamente en la biografía en cuestión, pero que figura en la contraposición entre Pericles y Fabio Máximo" (p. 158). Frente a lo que se ha visto hasta ahora, el período entre el 404 y el triunfo macedonio es interpretado por Plutarco en clave espartano y tebanocéntrica, evitando el contexto ateniense y apartándose tanto de la historiografía y la biografía de ascendencia eforea como de la propaganda imperial que insistía en la persistencia monumental de Atenas. La nueva amenaza macedonia y los prejuicios antimacedonios tienen una larga tradición antes de que Plutarco los recoja; pero se salva la figura de Alejandro, idealizada tanto en la biografía homónima como en De Alexandri Magni fortuna aut virtute. En cuanto a occidente, Plutarco se aleja de la tradición que igualaba, como victoria frente al bárbaro, la derrota de cartagineses y persas; en su obra están muy desdibujadas figuras como Gelón o
Hierón I y sólo dedica biografías a Dión y a Timoleonte, el primero claramente idealizado como paradigma del político-filósofo. La ausencia de una biografía de un personaje del peso de Dionisio I, explica Muccioli, se ve compensada por la serie de anécdotas sobre él que recorren la obra de Plutarco.

El capítulo 4, "Alessandro, l'ellenismo e la "fine della storia greca"' (pp. 193-253) arranca con la poderosa figura de Alejandro Magno. Para el autor, Alejandro se convierte en Plutarco en un "canon interpretativo": el tema de la imitatio Alexandri aparece en numerosas ocasiones en las vidas: Demetrio, Pirro, Pompeyo, César. De nuevo Muccioli atiende a las diferencias entre las Vitae y otras obras del corpus plutarqueo ya que, como señala, en De Alexandri Magni fortuna aut virtute la imagen es diferente a la que se traza en la biografía. El Alejandro de la primera es el personaje ecuménico, el único capaz de unir Oriente y Occidente. En la Vita, aparecen en mayor número las anécdotas, encaminadas a una construcción del personaje que conectara culturalmente con las expectativas y conocimientos de los pepaideumenoi destinatarios de la obra. Llama la atención Muccioli sobre la ausencia de comparatio al final de la pareja AlejandroCésar y sus posibles motivaciones. La historia griega de edad helenística y edad imperial, con la excepción de Alejandro, es vista por autores como Dionisio de Halicarnaso y Polibio como una época de decadencia, punto de vista al que se adhiere Plutarco. Hay otra figura, no obstante, que se salva: Foción, presentado con tintes claramente favorables $\mathrm{y}$ basados en fuentes muy tendenciosas. El período que sigue a la muerte de Foción es ignorado por Plutarco que pasa directamente a Demetrio, figura de la que destaca el papel político frente al legislador. En este capítulo Muccioli aborda también el asunto de la periodización histórica: frente a los historiadores que siguen el esquema de la 
sucesión de diferentes hegemonías nacionales, Plutarco tiene una perspectiva centrada en las ciudades, Esparta-Atenas-Tebas (hegemonía ateniense -vidas de Temístocles, Arístides, Cimón, Pericles y Alcibíades-, hegemonía espartana -vidas de Lisandro y Agesilao-, hegemonía tebana - Pelópidas y Epaminondas, perdida). El nexo entre historia griega e historia romana lo representa Flaminio, en cuya Vita se menciona la proclamación de la libertad de los griegos en el 196. Los reyes helenísticos son, en general, juzgados de manera negativa por Plutarco y esa edad helenística es abordada desde una perspectiva continental, con especial atención a los espartanos Agis IV y Cleómenes III, reyes reformadores en la estela de Licurgo.

En las páginas casi finales, "Plutarco tra storia, esempi e aneddoti. Quasi una conclusione" (pp. 255-259) se subrayan las características e intención de este ensayo: reconociendo que Plutarco se centra especialmente en la construcción ética de los personajes y deja el marco histórico, social y político bastante desdibujado, el autor ha realizado un gran esfuerzo por recuperar ese contexto y por definir el método histórico seguido. Se ha insistido, por ejemplo, en el uso diferente de las fuentes en las Vitae y en Moralia y en cómo Plutarco ha buscado construir un modelo de biografía, "un modelo que se confronta, explícitamente, no con la tradición biográfica precedente, sistemáticamente eludida o apenas mencionada, sino con la historiografia 'alta' y la erudición, también con la más especializada" (p. 257). Si a lo largo del libro han sido frecuentes las alusiones a los lectores "ideales" de Plutarco, de nuevo al final se vuelve a ellos para señalar que el de Queronea se esforzó por sintetizar conocimientos que formarían parte del bagaje cultural de los pepaideumenoi con innovaciones interpretativas, el uso de las anécdotas y un alejamiento de los modelos clá- sicos (Heródoto, Tucídides, Jenofonte) debido también al deseo de evitar redundancias.

$\mathrm{Si}$ al inicio Muccioli planteaba como objetivo de este ensayo hacerse una idea de cómo interpretaría la historia griega un erudito de época imperial, éstas serían, en conclusión, las coordenadas en las que se situaría: un spatium historicum que va de Heracles al último de los griegos, Filopemen, separado (cuando era posible, cosa que no siempre ocurría, véase la biografía de Teseo) del spatium mythicum $\mathrm{y}$ una predilección por personajes que pertenecen sobre todo a los momentos más brillantes de Atenas, Esparta y Tebas.

El libro se cierra con un apéndice, "Plutarco e il Ruler Cult nel mondo greco" (pp. 261-277) que aborda un tema muy espinoso para un griego, la bárbara costumbre del culto al soberano. De nuevo Muccioli señala el uso que hace de las fuentes Plutarco según le convenga insistir en unos o en otros aspectos de un personaje: en el caso de Alejandro, la pretensión de la proskynesis es criticada en De adulatore e amico y, por el contrario, enteramente eludida en De Alexandri Magni fortuna aut virtute.

Una completa bibliografía y un cuidado índice de pasajes citados cierran este estudio en el que se ha tratado con profundidad un tema tan complejo como el del método historiográfico de Plutarco. La extensión de la obra plutarquea y la inmensidad de la bibliografía al respecto, que podían haber sido dos graves escollos para llevar adelante la empresa, se solventan de una manera eficaz, eligiendo con precisión los pasajes que mejor podían ilustrar el argumento y sirviéndose de las citas a pie de página, muy numerosas pero muy precisas, de una manera ejemplar.

Marta GonzÁlez GonZÁlez Universidad de Málaga 